\title{
A smooth approximation for non-linear second order boundary value problems using composite non-polynomial spline functions
}

\author{
Anju Chaurasia, Yogesh Gupta and Prakash C. Srivastava
}

\begin{abstract}
A different amalgamation of non-polynomial splines is used to find the approximate solution of linear and non-linear second order boundary value problems. Cubic spline functions are assembled with exponential and trigonometric functions to develop the different orders of numerical schemes. Free parameter $k$ of the non-polynomial part is also used to form a new scheme, which elevates the accuracy of the solution. Numerical illustrations are given to validate the applicability and feasibility of the present methods and also depicted in the graphs.
\end{abstract}

Mathematics Subject Classification (2010): 41A15, 65D07, 65D15, 65L10.

Keywords: Cubic non-polynomial spline, second order boundary value problems, numerical approximation, error Analysis, convergence analysis.

\section{Introduction}

To demonstrate the basic concept and idea of our technique, we consider the following general non-linear second order two point boundary value problems (BVPs), which arise in a wide variety of engineering applications

$$
u^{(2)}(x)=f(x, u),-\infty \leqslant a \leqslant x \leqslant b \leqslant \infty
$$

with the boundary conditions (BCs)

$$
u(a)=A_{1}, u(b)=A_{2},
$$

where $A_{i}, \mathrm{i}=1,2$ are arbitrary finite real constants and $-\infty<u<\infty$. The function $f(x, u(x))$ is a continuous function of two variables with $f_{u} \geqslant 0$ on $[a, b]$. DE (1.1) with BC (1.2) has a unique solution, whose existence and uniqueness can be studied in [24]. For the linear case, $f(x, u)=p(x) u+g(x)$ with $p(x)$ and $g(x)$ continuous functions on the interval $[a, b]$. 
It is well acknowledged that numerous real-life phenomena in physics and engineering sciences often convert to boundary value problems for second order differential equations such as in heat transfer, optimal control, deflection in cables and plates, vibration of springs, electric circuits and in a number of other scientific applications [19]. Most of the BVPs are essentially solved using numerical approaches as those are not explained enough using existing analytical approaches. Consequently, some useful numerical schemes were being promoted, most notably spline-based' schemes. Spline functions were applied by many authors to establish the accurate and efficient numerical schemes for the solution of boundary value problems [4]. An exploration of the literature on a number of polynomial and non-polynomial spline techniques to solve the second order BVPs can be comprehended as quadratic spline method $[8,26$, 32, 42, 49], cubic spline method [2-3, 5, 9-12, 15, 20-23, 27-28, 30-34, 36-38, 40-41, 50], quartic spline method [6, 13-14, 29, 47], quintic spline method [7, 16, 43, 48] and others $[39,46]$. Voluminous research work have been contributed to this field but we are mainly concerned on those papers which have implemented non-polynomial splines for the solution of second order BVPs with various types of boundary conditions.

For instance, Rashidinia et al. [40] built up a technique based on cubic nonpolynomial spline functions of the form

$$
T_{n}=\operatorname{Span}\{1, x, \sin (\tau x), \cos (\tau x)\},
$$

They applied their scheme to acquire the numerical solution of the following form of second order two point BVPs

$$
-\frac{d}{d x}\left[p(x) \frac{d u}{d x}\right]=g(x) ; u(a)=u(b)=0 .
$$

Here, authors employed direct method to simplify the obtained system and facilitated the smooth approximations to linear second order BVPs. Similar approach was exercised by Islam and Tirmizi [27] to find the approximate solution of the system of two-point second order BVPs with Dirichlet BCs (1.2). They established the consistency equations to attain the desired results and solved linear second order equations to show the feasibility of their method. Khan and Aziz [34] proposed the parametric cubic spline functions with a parameter for attaining approximations to the solutions of the system of BVP. They presented improved results while comparing with some existing methods. Former approach [35] was yet again instituted by Khan in [33] to solve the following second order linear BVPs

$$
y^{(2)}(x)=f(x) y(x)+g(x) ; a \leqslant x \leqslant b
$$

with Dirichlet BCs (1.2). Here, the author developed the method of order four for specific values of parameters, or else his method was of order two. Over again, Zahra et al. [50] used cubic non-polynomial spline function space (1.3) to compute approximation to the solution of above linear BVPs (1.5) but with Neumann BCs. Kalyani and Rao [31] also adopted similar approach demonstrated by [27, 40, 50] to solve the following BVP of second order

$$
-\frac{d}{d x}\left[p(x) \frac{d u}{d x}\right]+v(x) u(x)=g(x) ; u(a)=u(b)=0 .
$$


They solved many linear and non-linear examples to study the performance of their method. Cubic non-polynomial spline scheme was once more deliberated by Justine and Sulaiman [30] to solve the general linear second order BVPs subject to Dirichlet BCs. To solve the obtained linear system, they used successive over relaxation in conjunction with Gauss-Seidel method. However, to establish the result, here authors considered the total number of iterations, execution times along with maximum absolute error (MAE).

Above, we have summarized numerous contributions that are made to deal with the solution of various types of second order BVPs choosing non-polynomial splines. The present research could contribute remarkably to this field as it includes some novel methods to solve non-linear second order BVPs with significant results. Our method is based on distinctive exponential and trigonometric spline function space given as

$$
\begin{gathered}
T_{3}=\operatorname{Span}\left\{1, x, e^{k x}, \sin (k x)\right\} \\
=\operatorname{Span}\left\{1, x,\left(\frac{2}{k^{2}}\right)\left(e^{k x}-k x-1\right),\left(\frac{6}{k^{3}}\right)(k x-\sin (k x))\right\},
\end{gathered}
$$

where $k$ is the frequency of trigonometric and exponential part of the spline function, which can be real or pure imaginary. It follows that if $k \rightarrow 0, T_{3}$ reduces to $\operatorname{Span}\left(1, x, x^{2}, x^{3}\right)$. In this paper, we have developed different order methods along with a modified $k$-dependent method based on the angular frequency of the nonpolynomial part for smooth approximation of the second order linear and non-linear BVPs. We have solved several examples using our developed methods and also shown comparisons of our results with some known methods like collocation, finite difference, Galerkin, Adomian decomposition and other spline methods. Our spline method solution and comparisons demonstrate that our algorithm performs comparatively better with more precise results.

Now, the paper is organized as follows: section 2 shows the formulation of our schemes and section 3 describes the solution of BVPs using the developed scheme. Section 4 deliberates the convergence of the schemes, while in section 5 some examples are solved using our developed spline methods. Paper is concluded in section 6 .

\section{Derivation of the method}

In this section, we develop a numerical method to approximate the solution of second order BVP (1.1)-(1.2). To do that, we first set a framework of $N+1$ equally spaced points $x_{i}$ of an interval [a, b] and divide them into $N$ equal sections such that $x_{i}=a+i h, i=0,1,2, \ldots, N$ where $x_{0}=a, x_{N}=b$ and $h=\frac{(b-a)}{N}$. Then, our spline function $P_{i}(x)$ holds the following structure in every section of the interval

$$
P_{i}(x)=a_{i} \sin k\left(x-x_{i}\right)+b_{i} e^{k\left(x-x_{i}\right)}+c_{i}\left(x-x_{i}\right)+d_{i} ; i=0,1,2 \ldots, N,
$$

where $a_{i}, b_{i}, c_{i}$ and $d_{i}$ are constants and $k$ is free parameter, which can be real or purely imaginary and will be used to raise the accuracy of the method. The function $P_{i}(x)$, which interpolates $S(x)$ at the mesh points $x_{i}$ and reduces to cubic spline as $k \rightarrow 0$, where $S(x)$ is the approximate solution of (1.1). Let $u(x)$ be the exact solution 
and $S_{i}$ be an approximation to $u_{i}=u\left(x_{i}\right)$ obtained by the segment $P_{i}(x)$ of the spline function passing through the points $\left(x_{i}, S_{i}\right)$ and $\left(x_{i+1}, S_{i+1}\right)$. Then the mixed spline defined by the function $S(x)=P_{i}(x)$.

Now, we assume

$$
P_{i}\left(x_{i}\right)=S_{i}, \quad P_{i}\left(x_{i+1}\right)=S_{i+1}, \quad P_{i}^{(2)}\left(x_{i}\right)=M_{i}, \quad P_{i}^{(2)}\left(x_{i+1}\right)=M_{i+1},
$$

to get the following value of coefficients

$$
\begin{gathered}
a_{i}=\frac{1}{k^{2} \sin (\theta)}\left[e^{\theta} M_{i}-M_{i+1}\right], \quad b_{i}=\frac{1}{k^{2}}\left[M_{i}\right], \\
c_{i}=\frac{S_{i+1}-S_{i}}{h}+\frac{M_{i+1}+M_{i}}{k^{2} h}-\frac{2 e^{\theta} M_{i}}{k^{2} h}, \quad d_{i}=S_{i}-\frac{1}{k^{2}}\left[M_{i}\right],
\end{gathered}
$$

whereby $\theta=k h$ and $i=0,1,2, \ldots ., N$.

Next, use the continuity condition of the first derivative and substitute the value of coefficients $a_{i}, b_{i}, c_{i}$ and $d_{i}$. After some algebraic manipulations, we can obtain the following main relation

$$
S_{i-1}-2 S_{i}+S_{i+1}=h^{2}\left[\alpha M_{i-1}+\beta M_{i}+\gamma M_{i+1}\right] ; i=1,2, \ldots N-1,
$$

where,

$$
\begin{gathered}
\alpha=\frac{\theta e^{\theta}\{\sin (\theta)+\cos (\theta)\}+\sin (\theta)\left(1-2 e^{\theta}\right)}{\theta^{2} \sin (\theta)}, \\
\beta=\frac{2 e^{\theta} \sin (\theta)-\theta e^{\theta}-\theta\{\sin (\theta)+\cos (\theta)\}}{\theta^{2} \sin (\theta)}, \\
\gamma=\frac{\theta-\sin (\theta)}{\theta^{2} \sin (\theta)}
\end{gathered}
$$

and $M_{i}=S^{(2)}\left(x_{i}\right)=f(x, u)$, by discretizing the considered DE (1.1) at the nodal point $x_{i}$. As $k \rightarrow 0, \alpha=1 / 6, \beta=4 / 6$ and $\gamma=1 / 6$, our scheme (2.2) reduces to ordinary cubic spline scheme [5] and then, it is evidently second order convergent.

Accordingly, equation (2.2) provides a system of $N-1$ non-linear algebraic equations in the $N-1$ unknowns $S_{i}, i=1,2, \ldots, N-1$, which by discretizing can be written as

$$
\left(S_{i-1}-\alpha h^{2} f\left(x_{i-1}, S_{i-1}\right)\right)-\left(2 S_{i}+\beta h^{2} f\left(x_{i}, S_{i}\right)\right)+\left(S_{i+1}-\gamma h^{2} f\left(x_{i+1}, S_{i+1}\right)\right)+t_{i}=0 .
$$

Then, the local truncation error $t_{i}, i=1,2, \ldots, N-1$, can be written as

$$
\begin{aligned}
t_{i} & =\{1-(\alpha+\beta+\gamma)\} h^{2} u_{i}^{(2)}+(\alpha-\gamma) h^{3} u_{i}^{(3)}+\left\{\frac{1}{12}-\frac{1}{2}(\alpha+\gamma)\right\} h^{4} u_{i}^{(4)} \\
& +\frac{1}{6}(\alpha-\gamma) h^{5} u_{i}^{(5)}+\left\{\frac{1}{360}-\frac{1}{24}(\alpha+\gamma)\right\} h^{6} u_{i}^{(6)}+O\left(h^{7}\right) .
\end{aligned}
$$

Thus, our schemes (2.2) and (2.4) give rise to a family of methods of different orders as follows: 


\subsection{Different order of methods}

Case (i). First order method

For $\alpha+\beta+\gamma=1, \alpha \neq \gamma$. Here,

$$
\begin{gathered}
t_{i}=(\alpha-\gamma) h^{3} u_{i}^{(3)}+O\left(h^{4}\right), \\
\|T\|=|(\alpha-\gamma)| h^{3} M_{3}, M_{3}=\max \left|u^{(3)}(x)\right| .
\end{gathered}
$$

Case (ii). Second order method

For $\alpha+\beta+\gamma=1, \alpha=\gamma$ and $\alpha+\gamma \neq \frac{1}{6}$. Here,

$$
\begin{gathered}
t_{i}=\left\{\frac{1}{12}-\frac{1}{2}(\alpha+\gamma)\right\} h^{4} u_{i}^{(4)}+O\left(h^{5}\right), \\
\|T\|=\left|\frac{1}{12}-\frac{1}{2}(\alpha+\gamma)\right| h^{4} M_{4}, M_{4}=\max \left|u^{(4)}(x)\right| .
\end{gathered}
$$

Case (iii). Fourth order method

For $\alpha+\beta+\gamma=1, \alpha=\gamma$ and $\alpha+\gamma=\frac{1}{6}$. Here,

$$
\begin{gathered}
t_{i}=\left\{\frac{1}{360}-\frac{1}{24}(\alpha+\gamma)\right\} h^{6} u_{i}^{(6)}+O\left(h^{7}\right), \\
\|T\|=\left|\frac{1}{360}-\frac{1}{24}(\alpha+\gamma)\right| h^{6} M_{6}, M_{6}=\max \left|u^{(6)}(x)\right| .
\end{gathered}
$$

where $\|\cdot\|$ represents the $\infty$ norm in matrix vector.

\subsection{Modified $k$-dependent method}

In this section, we will use the parameter $k$ to raise the order of accuracy of the obtained scheme (2.2). To do this, we first rearrange the terms in equation (2.4) in the following manner

$$
\begin{aligned}
t_{i} & =h^{4}\left[\frac{1}{\theta^{2}}+\frac{\left(e^{\theta}-1\right)(1-\cos (\theta))+\sin (\theta)\left(1+e^{\theta}\right)}{\theta^{3} \sin (\theta)}\right]\left(k^{2} u_{i}^{(2)}-u_{i}^{(4)}\right) \\
& +h^{5}\left[\frac{e^{\theta}(\sin (\theta)+\cos (\theta))-1}{\theta^{3} \sin (\theta)}+\frac{2\left(1-e^{\theta}\right)}{\theta^{4}}\right] k^{2} u_{i}^{(3)} \\
& +h^{6}\left[\frac{1}{12 \theta^{2}}-\frac{1+e^{\theta}(\sin (\theta)+\cos (\theta))}{2 \theta^{3} \sin (\theta)}+\frac{\left(1+e^{\theta)}\right.}{\theta^{4}}\right] k^{2} u_{i}^{(4)} \\
& +h^{6}\left[\frac{(\sin (\theta)+\cos (\theta))+1+e^{\theta}(\sin (\theta)-\cos (\theta)-1)}{\theta^{5} \sin (\theta)}\right] k^{2} u_{i}^{(4)} \\
& +h^{6}\left[\left\{\frac{1}{360}+\frac{-e^{\theta}(\sin (\theta)+\cos (\theta))}{24 \theta \sin (\theta)}+\frac{\left(2 e^{\theta}-1\right)}{24 \theta^{2}}\right\} u_{i}^{(6)}\left(\eta_{1}\right)+\left\{\frac{1}{24 \theta^{2}}-\frac{1}{24 \theta \sin (\theta)}\right\} u_{i}^{(6)}\left(\eta_{2}\right)\right] \\
& +h^{7}\left[\frac{e^{\theta}(\sin (\theta)+\cos (\theta)-1)}{6 \theta^{3} \sin (\theta)}+\frac{\left(1-e^{\theta}\right)}{3 \theta^{4}}\right] k^{2} u_{i}^{(5)}+\cdots
\end{aligned}
$$

Equating the coefficient of the leading term in the above equation to zero, we can get the equation in $k_{i}$ as

$$
k_{i}^{2}=\frac{u_{i}^{(4)}}{u_{i}^{(2)}}=\frac{f^{\prime \prime}\left(x_{i}, u_{i}\right)}{f\left(x_{i}, u_{i}\right)}
$$


For the linear case, $f\left(x_{i}, u_{i}\right)=p_{i} u_{i}+g_{i}$. Then,

$$
k_{i}^{2}=\frac{\left(p_{i}^{\prime \prime}+p_{i}^{2}\right) u_{i}+2 p_{i}^{\prime} u_{i}^{\prime}+p_{i} g_{i}+g_{i}^{\prime \prime}}{p_{i} u_{i}+g_{i}}
$$

Thus, from above we see that calculation of $k_{i}$ requires the approximations for $u_{i}$ and $u_{i}^{\prime}$. Approximation for $u_{i}$ can be obtained by means of our developed scheme (2.2) for $k=0$ and for $u_{i}^{\prime}$, following steps can be adapted:

(i) Differentiating equation $(2.1)$ at $x=x_{i}$, to get

$$
\begin{aligned}
P_{i}^{\prime}(x) & =\frac{1}{k \sin (\theta)}\left\{\left(\sin (\theta)+e^{\theta}\right) M_{i}-M_{i+1}\right\} \\
& +\frac{\left(S_{i+1}-S_{i}\right)}{h}+\frac{1}{k^{2} h}\left\{\left(1-2 e^{\theta}\right) M_{i}+M_{i+1}\right\},
\end{aligned}
$$

(ii) If the limit $k$ going to zero in the above equation, we obtain

$$
P_{i}^{\prime}(x)=-\frac{h}{6} f\left(x_{i+1}, u_{i+1}\right)-\frac{h}{3} f\left(x_{i}, u_{i}\right)+\frac{\left(S_{i+1}-S_{i}\right)}{h} ; i=0,1, \ldots ., N .
$$

\section{Composite non-polynomial spline solution}

To develop the approximation to the solution of BVP (1.1)-(1.2) based on our developed spline method, we write our scheme (2.2) in the following standard matrix form:

$$
A_{0} S^{(1)}-h^{2} B f^{(1)}\left(S^{(1)}\right)=C^{(1)}
$$

where $A_{0}$ and $B$ are three-band square matrices of order $N-1$, given by

$$
\begin{aligned}
A_{0} & =\left[\begin{array}{cccccccccc}
-2 & 1 & & & & & & & & \\
1 & -2 & 1 & & & & & & & \\
& 1 & -2 & 1 & & & & & & \\
& \ldots & \ldots & \ldots & \ldots & \ldots & \ldots & \ldots & \ldots & \ldots \\
& \ldots & \ldots & \ldots & \ldots & \ldots & \ldots & \ldots & \ldots & \ldots \\
& & & & & & & 1 & -2 & 1 \\
& & & & & & & & 1 & -2
\end{array}\right] \\
B & =\left[\begin{array}{cccccccccc}
\beta & \gamma & & & & & & & & \\
\alpha & \beta & \gamma & & & & & & & \\
& \alpha & \beta & \gamma & & & & & & \\
& \ldots & \ldots & \ldots & \ldots & \ldots & \ldots & \ldots & \ldots & \ldots \\
& \ldots & \ldots & \ldots & \ldots & \ldots & \ldots & \ldots & \ldots & \ldots \\
& & & & & & & \alpha & \beta & \gamma \\
& & & & & & & & \alpha & \beta
\end{array}\right]
\end{aligned}
$$

Matrix: $f^{(1)}\left(S^{(1)}\right)=f\left(x_{i}, S_{i}^{(1)}\right), S^{(1)}=\left[S_{1}, S_{2}, \ldots, S_{N-1}\right]^{t}$ and

$$
C^{(1)}=\left\{\begin{array}{cl}
-A_{1}+h^{2} \alpha f\left(x_{0}, A_{1}\right), & i=1, \\
0, & i=2,3, \ldots N-2, \\
-A_{2}+h^{2} \gamma f\left(x_{N}, A_{2}\right), & N-1 .
\end{array}\right.
$$


Likewise,

$$
A_{0} U^{(1)}-h^{2} B f^{(1)}\left(U^{(1)}\right)=C^{(1)}+T^{(1)},
$$

where the vector $U^{(1)}=u\left(x_{i}\right)$ is the exact solution with truncation error $T^{(1)}=\left(t_{i}^{(1)}\right)$, for $i=1,2, \ldots, N-1$.

From (3.1) and (3.2), we have

$$
\left[A_{0}-h^{2} B Q\right] E^{(1)}=T^{(1)}
$$

where

$$
E^{(1)}=U^{(1)}-S^{(1)}=\left[e_{1}^{(1)}, e_{2}^{(1)}, \ldots \ldots, e_{N-1}^{(1)}\right]^{T}
$$

and

$$
Q=\operatorname{diag}\left(\frac{\partial f_{i}^{(1)}}{\partial u_{i}^{(1)}}\right), i=1,2, \ldots, N-1
$$

is the diagonal matrix of order $N-1$, whereas for the linear case, $\mathrm{Q}=\operatorname{diag}\left(f_{i}^{(1)}\right)$.

Thus, the equations (3.1)-(3.3) demonstrate our scheme, using which one can obtain the approximate solution of non-linear DE (1.1) with the BC (1.2). We shall use Newton's method to obtain the solution of the non-linear system (2.2), which converge to the solution of (1.1)-(1.2) for all sufficiently small values of $\mathrm{h}[24,46]$.

\section{Convergence analysis}

Now, we will derive a bound on $\left\|E^{(1)}\right\|$. From equation (3.3), we get

$$
A E^{(1)}=T^{(1)},
$$

where, $\mathrm{A}=\left[A_{0}-h^{2} B Q\right]$ is a tri-diagonal matrix. The elements of $\mathrm{A}$ are given by

$$
a_{i j}=\left\{\begin{array}{cl}
-2-h^{2} \beta f_{u}\left(x_{i}, u_{i}\right), & i=j \\
1-h^{2} \alpha f_{u}\left(x_{i}, u_{i}\right), & i-j=1 \\
1-h^{2} \gamma f_{u}\left(x_{i}, u_{i}\right), & j-i=1 \\
0, & |i-j|>1
\end{array}\right.
$$

From above, we have

$$
\left\|E^{(1)}\right\| \leq\left\|A^{-1}\right\|\left\|T^{(1)}\right\|
$$

(See [24]) $\left\|A^{-1}\right\| \leq(b-a)^{2} / 8 h^{2}$ and so, we can infer the following convergent schemes:

\section{Case 4.1. First order convergent method}

$$
\text { For }(\alpha, \beta, \gamma)=(75 / 1920,1755 / 1920,90 / 1920),\left\|T^{(1)}\right\|_{\infty}=\frac{1}{128} h^{3} M_{3} \text {. }
$$

Then from equation $(2.5)$, we get

$$
\left\|E^{(1)}\right\| \leq K_{1} h \cong O\left(h^{1}\right) .
$$

This relation (4.1) shows that the method is first order convergent.

\section{Case 4.2. Second order convergent method}

$$
\text { For } \alpha=\gamma=\frac{3}{38} \text { and } \beta=\frac{32}{38},\left\|T^{(1)}\right\|_{\infty}=\frac{1}{128} h^{4} M_{4} \text {. }
$$


Then it follows from (2.6) that

$$
\left\|E^{(1)}\right\| \leq K_{2} h^{2} \cong O\left(h^{2}\right)
$$

The relation (4.2) confirms second order convergence of the method.

\section{Case 4.3. Fourth order convergent method}

For $\alpha=\gamma=\frac{1}{12}$ and $\beta=\frac{10}{12},\left\|T^{(1)}\right\|_{\infty}=\frac{1}{240} h^{6} M_{6}$.

Then from equation (2.7), we have

$$
\left\|E^{(1)}\right\| \leq K_{3} h^{4} \cong O\left(h^{4}\right)
$$

which confirms fourth order convergence of the method.

\section{Numerical illustration}

To illuminate the use of our developed methods, we have considered several linear and non-linear examples of second order BVPs and also compared our results with other existing methods.

Problem 5.1. Consider the linear BVP

$$
u^{(2)}(x)=\frac{2}{x^{2}} u-\frac{1}{x} ; \quad 2<x<3 ; \quad u(2)=u(3)=0 .
$$

The theoretical (exact) solution of (5.1) is

$$
u(x)=\frac{1}{38}\left(-5 x^{2}+19 x-\frac{36}{x}\right) .
$$

Comparing the given equation (5.1) with (1.1) at $x=x_{i}$, we have

$$
f\left(x_{i}, u_{i}\right)=\frac{2}{x_{i}^{2}} u_{i}-\frac{1}{x_{i}} .
$$

Table 1. Absolute error for the solution of Problem 5.1 at different value of $x$ for $N=8$

\begin{tabular}{cccc}
\hline$x$ & Our method for $k=0$ & Our $k$-based method & Value of $k$ \\
\hline $17 / 8$ & $2.36 \times 10^{-5}$ & $4.28 \times 10^{-6}$ & 1.0674 \\
$18 / 8$ & $3.66 \times 10^{-5}$ & $6.31 \times 10^{-6}$ & 0.9581 \\
$19 / 8$ & $4.16 \times 10^{-5}$ & $6.86 \times 10^{-6}$ & 0.8623 \\
$20 / 8$ & $4.07 \times 10^{-5}$ & $6.45 \times 10^{-6}$ & 0.7781 \\
$21 / 8$ & $3.52 \times 10^{-5}$ & $5.38 \times 10^{-6}$ & 0.7040 \\
$22 / 8$ & $2.61 \times 10^{-5}$ & $3.87 \times 10^{-6}$ & 0.6387 \\
$23 / 8$ & $1.42 \times 10^{-5}$ & $2.05 \times 10^{-6}$ & 0.5809 \\
\hline
\end{tabular}

For the linear case, $f(x, u)=p(x) u+g(x)$, so $p_{i}=p\left(x_{i}\right)=2 / x_{i}^{2} ; g_{i}=g\left(x_{i}\right)=-1 / x_{i}$ and equation(3.1) is changed to $A S=C$, where $A=A_{0}-h^{2} B Q ; Q=\operatorname{diag}\left(f_{i}\right)$. By substituting these values, we get system of linear equations for Problem 5.1 that can be solved using any suitable method. Absolute errors at different point of $x$ are summarized in Table 1 for $k=0$, i.e. $(\alpha, \beta, \gamma)=(1 / 6,4 / 6,1 / 6)$ and $k$-based method, when $h=1 / 8$. Results indicate that the modified $k$-dependent method provides better 
results than the method for $k=0$. The value of parameter $k$ at different value of $x$ is also listed in Table 1 (col. IV).

Table 2 reports the MAE at different value of $N$ for second order schemes together with $k$-based technique. Table indicates that $k$-based method is a third order convergent method. Comparison of numerical results with other existing methods is also included in this table. Fourth order method solution when $(\alpha, \beta, \gamma)=(1 / 12$, 10/12, 1/12) of Problem 5.1 for $N=10$ is presented in Table 3, along with comparison with Galerkin method.

Table 2. Comparison of maximum absolute errors for Problem 5.1

\begin{tabular}{lccc}
\hline \multicolumn{1}{c}{ Our method } & $\mathrm{N}=4$ & $\mathrm{~N}=8$ & $\mathrm{~N}=16$ \\
\hline Our second order methods & & & \\
$(\alpha=\gamma=3 / 38, \beta=32 / 38)$ & $5.94 \times 10^{-6}$ & $2.00 \times 10^{-6}$ & $5.37 \times 10^{-7}$ \\
$(\alpha=\gamma=1 / 13, \beta=11 / 13)$ & $9.88 \times 10^{-6}$ & $3.01 \times 10^{-6}$ & $7.90 \times 10^{-7}$ \\
Our method for $k=0$ & $1.65 \times 10^{-4}$ & $4.16 \times 10^{-5}$ & $1.04 \times 10^{-5}$ \\
Our $k$-based Method & $5.05 \times 10^{-5}$ & $6.86 \times 10^{-6}$ & $8.61 \times 10^{-7}$ \\
Quadratic spline [9] & $1.60 \times 10^{-4}$ & $2.66 \times 10^{-5}$ & $5.58 \times 10^{-6}$ \\
Centered Difference method [10] & $2.79 \times 10^{-4}$ & $5.42 \times 10^{-5}$ & $1.19 \times 10^{-5}$ \\
Quadratic spline [42] & $7.93 \times 10^{-5}$ & $2.06 \times 10^{-5}$ & $5.20 \times 10^{-6}$ \\
Cubic spline [10] & $5.49 \times 10^{-5}$ & $1.87 \times 10^{-5}$ & $5.07 \times 10^{-6}$ \\
Cubic non-poly. spline [33] & $2.05 \times 10^{-5}$ & $5.74 \times 10^{-6}$ & $1.47 \times 10^{-6}$ \\
Discrete cubic spline [21] & $1.77 \times 10^{-5}$ & $5.00 \times 10^{-6}$ & $1.29 \times 10^{-6}$ \\
\hline
\end{tabular}
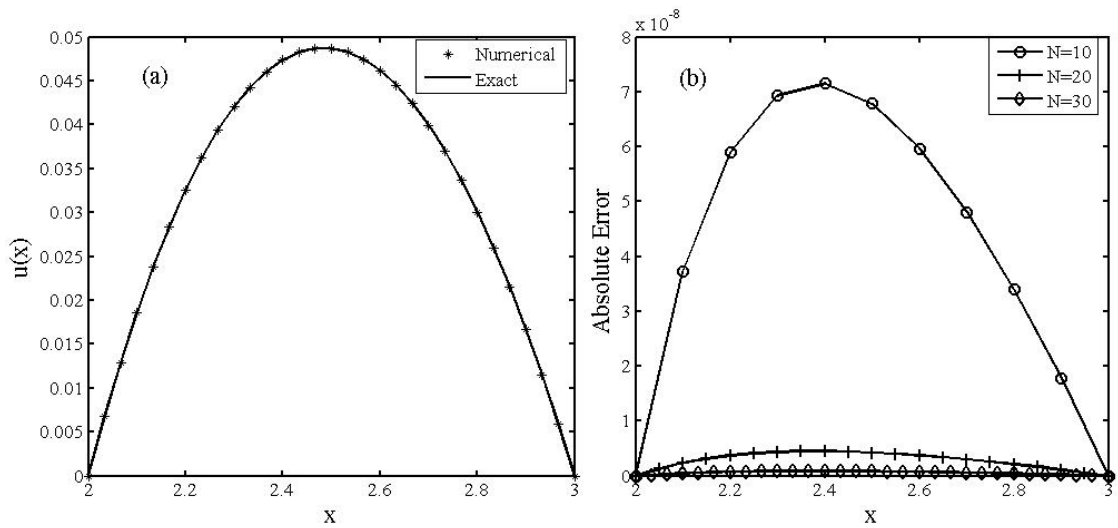

Figure 1. (a) Comparison of approximate and exact values for Problem 5.1.

(b) Error graph for Problem 5.1 at different values of N (Table 3). 
Table 3. Comparison of MAE for the solution of Problem 5.1

(Fourth order method)

\begin{tabular}{cccccc}
\hline$x$ & 2.1 & 2.2 & 2.3 & 2.4 & 2.5 \\
\hline Our method & $3.73 \times 10^{-8}$ & $5.89 \times 10^{-8}$ & $6.92 \times 10^{-8}$ & $7.15 \times 10^{-8}$ & $6.78 \times 10^{-8}$ \\
Galerkin method [25] & $2.52 \times 10^{-7}$ & $1.15 \times 10^{-6}$ & $6.73 \times 10^{-7}$ & $6.90 \times 10^{-7}$ & $1.24 \times 10^{-6}$ \\
\hline$x$ & 2.6 & 2.7 & 2.8 & 2.9 & \\
\hline Our method & $5.96 \times 10^{-8}$ & $4.81 \times 10^{-8}$ & $3.39 \times 10^{-8}$ & $1.77 \times 10^{-8}$ & \\
Galerkin method [25] & $4.51 \times 10^{-7}$ & $7.90 \times 10^{-7}$ & $9.70 \times 10^{-7}$ & $3.17 \times 10^{-7}$ & \\
\hline
\end{tabular}
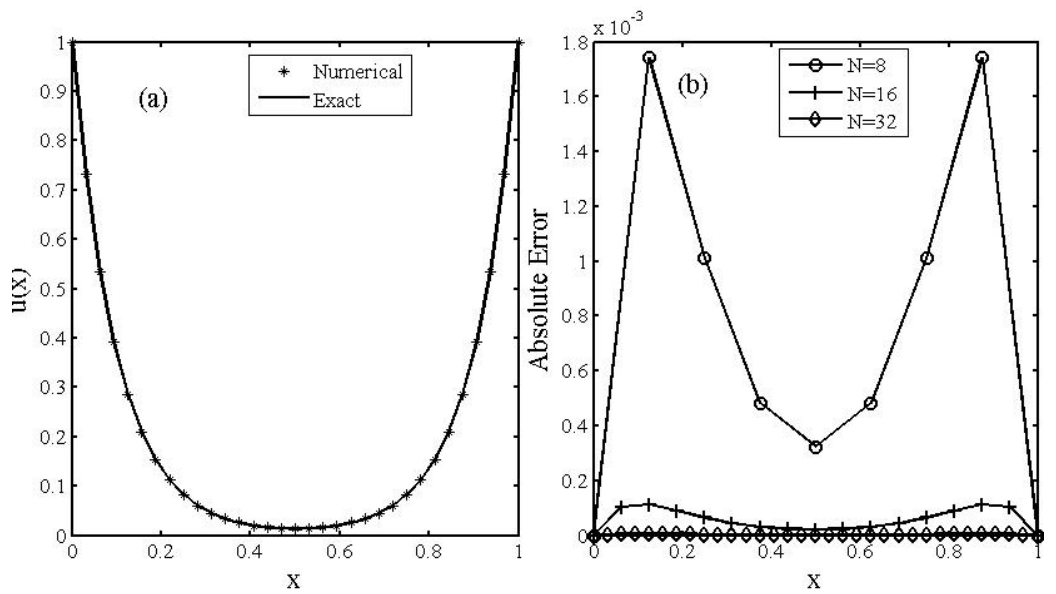

Figure 2. (a) Comparison of approximate and exact values for Problem 5.2.

(b) Error graph for Problem 5.2 at different values of N (Table 4).

Problem 5.2. Consider the linear BVP

$$
u^{(2)}(x)=100 u ; \quad 0<x<1 ; \quad u(0)=u(1)=1 .
$$

The theoretical solution of (5.3) is

$$
u(x)=\frac{\cosh (10 x-5)}{\cosh 5} .
$$

Problem 5.3. Consider the linear BVP

$$
u^{(2)}(x)=u+\cos (x), \quad 0<x<1 ; \quad u(0)=u(1)=1 .
$$

The theoretical solution of (5.5) is

$$
\begin{aligned}
u(x) & =\frac{-3 \cosh (1)+3 \sinh (1)+\cos (1)+2}{4 \sinh (1)} e^{x} \\
& +\frac{3 \cosh (1)+3 \sinh (1)-\cos (1)-2}{4 \sinh (1)} e^{-x}-\frac{\cos (x)}{2}
\end{aligned}
$$


Table 4. Comparison of maximum absolute errors for Problem 5.2

\begin{tabular}{lcccc}
\hline \multicolumn{1}{c}{ Our method } & $\mathrm{N}=16$ & $\mathrm{~N}=32$ & $\mathrm{~N}=20$ & $\mathrm{~N}=40$ \\
\hline$\alpha=\gamma=3 / 38, \beta=32 / 38$ & $1.95 \times 10^{-4}$ & $7.15 \times 10^{-5}$ & $1.54 \times 10^{-4}$ & $4.75 \times 10^{-5}$ \\
$\alpha=\gamma=1 / 13, \beta=11 / 13$ & $3.37 \times 10^{-4}$ & $1.07 \times 10^{-4}$ & $2.47 \times 10^{-4}$ & $7.08 \times 10^{-5}$ \\
Our method for $k=0$ & $6.10 \times 10^{-3}$ & $1.50 \times 10^{-3}$ & $3.90 \times 10^{-3}$ & $9.65 \times 10^{-4}$ \\
Our k-based method & $1.16 \times 10^{-2}$ & $1.11 \times 10^{-3}$ & $5.40 \times 10^{-3}$ & $5.57 \times 10^{-4}$ \\
Our fourth-order method & $1.12 \times 10^{-4}$ & $7.28 \times 10^{-6}$ & $4.75 \times 10^{-5}$ & $2.99 \times 10^{-6}$ \\
Cubic non-poly. spline [33] & $7.22 \times 10^{-4}$ & $2.06 \times 10^{-4}$ & $5.00 \times 10^{-4}$ & $1.34 \times 10^{-4}$ \\
Discrete cubic spline [21] & $6.18 \times 10^{-4}$ & $1.80 \times 10^{-4}$ & $4.32 \times 10^{-4}$ & $1.17 \times 10^{-4}$ \\
Quadratic spline [42] & $3.06 \times 10^{-3}$ & $7.58 \times 10^{-4}$ & - & - \\
Collocation method [32] & - & - & $1.80 \times 10^{-3}$ & $4.70 \times 10^{-4}$ \\
Cubic spline [10] & $2.27 \times 10^{-3}$ & $6.84 \times 10^{-4}$ & $1.57 \times 10^{-3}$ & $4.53 \times 10^{-4}$ \\
\hline
\end{tabular}

Maximum absolute errors at the different values of $N$ are tabulated in Table 4 for Problem 5.2 and in Table 5 for Problem 5.3. Fourth order method solution and error graphs at different values of $N$ are also given in Figures 1-3 respectively for Problems 5.1-5.3.

Table 5. Comparison of maximum absolute errors for the solution of Problem 5.3

\begin{tabular}{llllllll}
\hline & $\begin{array}{c}\text { Our } \\
\text { method } \\
\text { for } k=0\end{array}$ & $\begin{array}{c}\text { Our } \\
k \text {-based } \\
\text { method }\end{array}$ & $\begin{array}{l}\text { Our } \\
\text { fourth } \\
\text { order } \\
\text { method }\end{array}$ & $\begin{array}{l}\text { Standard } \\
\text { Tau- } \\
\text { method } \\
{[45]}\end{array}$ & $\begin{array}{l}\text { Perturbed } \\
\text { Tau- } \\
\text { method } \\
{[45]}\end{array}$ & $\begin{array}{l}\text { EADM } \\
{[17]}\end{array}$ & $\begin{array}{l}\text { EFM } \\
{[44]}\end{array}$ \\
\hline $1 / 8$ & $5.24 \times 10^{-4}$ & $7.13 \times 10^{-6}$ & $8.97 \times 10^{-8}$ & $1.00 \times 10^{-4}$ & $2.10 \times 10^{-4}$ & $4.37 \times 10^{-7}$ & $6.88 \times 10^{-5}$ \\
$2 / 8$ & $9.69 \times 10^{-4}$ & $1.17 \times 10^{-5}$ & $1.50 \times 10^{-7}$ & 0 & $1.10 \times 10^{-4}$ & $8.07 \times 10^{-7}$ & $4.93 \times 10^{-5}$ \\
$3 / 8$ & $1.26 \times 10^{-3}$ & $1.43 \times 10^{-5}$ & $1.84 \times 10^{-7}$ & $1.00 \times 10^{-4}$ & $7.51 \times 10^{-5}$ & $1.05 \times 10^{-6}$ & $3.21 \times 10^{-5}$ \\
$4 / 8$ & $1.37 \times 10^{-3}$ & $1.50 \times 10^{-5}$ & $1.93 \times 10^{-7}$ & $1.00 \times 10^{-4}$ & $6.25 \times 10^{-5}$ & $1.14 \times 10^{-6}$ & $2.63 \times 10^{-5}$ \\
$5 / 8$ & $1.26 \times 10^{-3}$ & $1.39 \times 10^{-5}$ & $1.79 \times 10^{-7}$ & $2.00 \times 10^{-4}$ & $4.31 \times 10^{-5}$ & $1.05 \times 10^{-6}$ & $2.16 \times 10^{-5}$ \\
$6 / 8$ & $9.69 \times 10^{-4}$ & $1.11 \times 10^{-5}$ & $1.42 \times 10^{-7}$ & $2.00 \times 10^{-4}$ & $2.43 \times 10^{-5}$ & $8.07 \times 10^{-7}$ & $1.09 \times 10^{-5}$ \\
$7 / 8$ & $5.24 \times 10^{-4}$ & $6.56 \times 10^{-6}$ & $8.32 \times 10^{-8}$ & $2.00 \times 10^{-4}$ & $1.13 \times 10^{-5}$ & $4.37 \times 10^{-7}$ & $1.01 \times 10^{-5}$ \\
\hline
\end{tabular}

Abbreviations: EADM: Extended Adomian Decomposition Method; EFM: Exponential fitting method
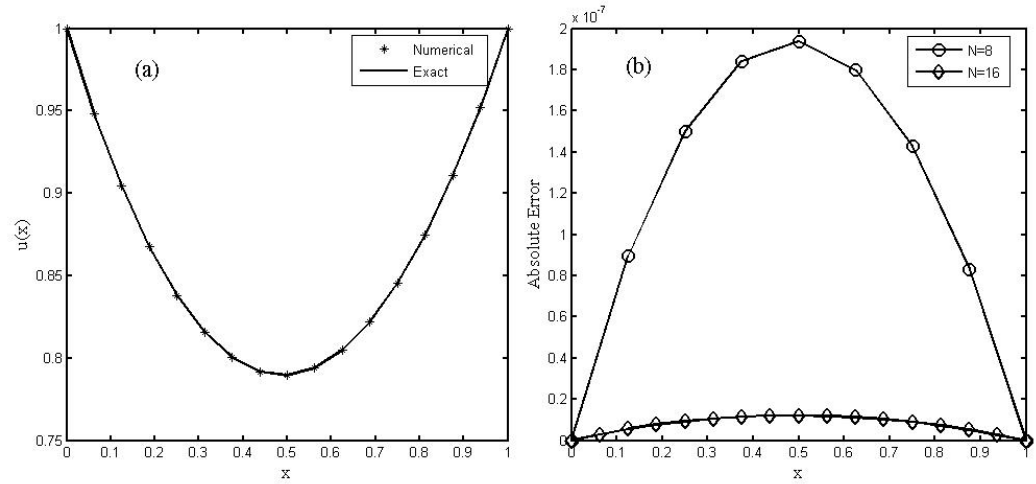

Figure 3. (a) Comparison of approximate and exact values for Problem 5.3.

(b) Error graph for Problem 5.3 at different values of N (Table 5). 
Problem 5.4. Consider the non-linear BVP

$$
u^{(2)}(x)=2(u(x))^{3}, \quad-1<x<0 ; \quad u(-1)=1 / 2, u(0)=1 / 3 .
$$

The theoretical solution of equation (5.7) is

$$
u(x)=\frac{1}{(x+3)}
$$

To solve non-linear BVP (Problem 5.4), compare the equation (5.7) with equation (1.1) at $x=x_{i}$ and we have

$$
f\left(x_{i}, u_{i}\right)=2\left(u\left(x_{i}\right)\right)^{3}
$$

Using equation (3.1), we obtain a system of non-linear equations that have been solved using Newton's method. Results are verified with MATLAB builtin solver(fsolve) command. Tables 6 and 7 show the maximum absolute errors, in case of $k=0$, modified $k$-dependent method and fourth order method solution. Tables clearly indicate that our developed methods produce the better accuracy than some other specified methods. We have also listed the value of parameter $k$ at different value of $x$ in Table 8 .

Table 6. Comparison of MAE at $\mathrm{N}=10$ for the solution of Problem 5.4

\begin{tabular}{clllll}
\hline $\begin{array}{c}\text { Our } \\
\text { method } \\
\text { for } k=0\end{array}$ & $\begin{array}{l}\text { Our } \\
k \text {-based } \\
\text { method }\end{array}$ & $\begin{array}{l}\text { Our fourth } \\
\text { order } \\
\text { method }\end{array}$ & $\begin{array}{l}\text { Quintic } \\
\text { spline [7] }\end{array}$ & $\begin{array}{l}\text { Cubic } \\
\text { spline[20] }\end{array}$ & $\begin{array}{l}\text { Quartic } \\
\text { spline [6] }\end{array}$ \\
\hline $2.65 \times 10^{-5}$ & $8.08 \times 10^{-6}$ & $3.23 \times 10^{-7}$ & $8.82 \times 10^{-6}$ & $1.68 \times 10^{-5}$ & $4.67 \times 10^{-6}$ \\
\hline
\end{tabular}

Table 7. Maximum absolute errors at different value of $N$ for Problem 5.4

\begin{tabular}{lccc}
\hline \multicolumn{1}{c}{ Our method } & $\mathrm{N}=4$ & $\mathrm{~N}=8$ & $\mathrm{~N}=16$ \\
\hline Our method for $k=0$ & $1.63 \times 10^{-4}$ & $4.13 \times 10^{-5}$ & $1.03 \times 10^{-5}$ \\
Our $k$-based method & $1.28 \times 10^{-4}$ & $1.53 \times 10^{-5}$ & $6.83 \times 10^{-6}$ \\
Our fourth-order method & $2.56 \times 10^{-6}$ & $1.64 \times 10^{-7}$ & $1.08 \times 10^{-8}$ \\
\hline
\end{tabular}

Table 8. The value of $k$ at different value of $x$ for the solution of Problem 5.4

\begin{tabular}{cccccccccc}
\hline$x$ & -0.9 & -0.8 & -0.7 & -0.6 & -0.5 & -0.4 & -0.3 & -0.2 & -0.1 \\
\hline$k$ & 1.6499 & 1.5748 & 1.5062 & 1.4433 & 1.3855 & 1.3321 & 1.2827 & 1.2368 & 1.1941 \\
\hline
\end{tabular}



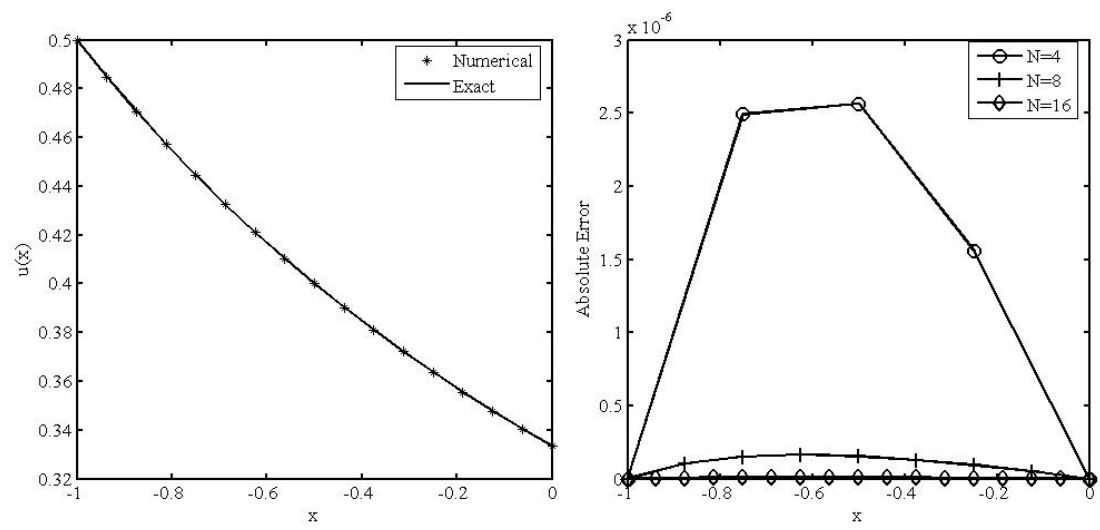

Figure 4. (a) Comparison of approximate values and exact values for Problem 5.4. (b) Error graph for Problem 5.4 at different values of N (Table 7).
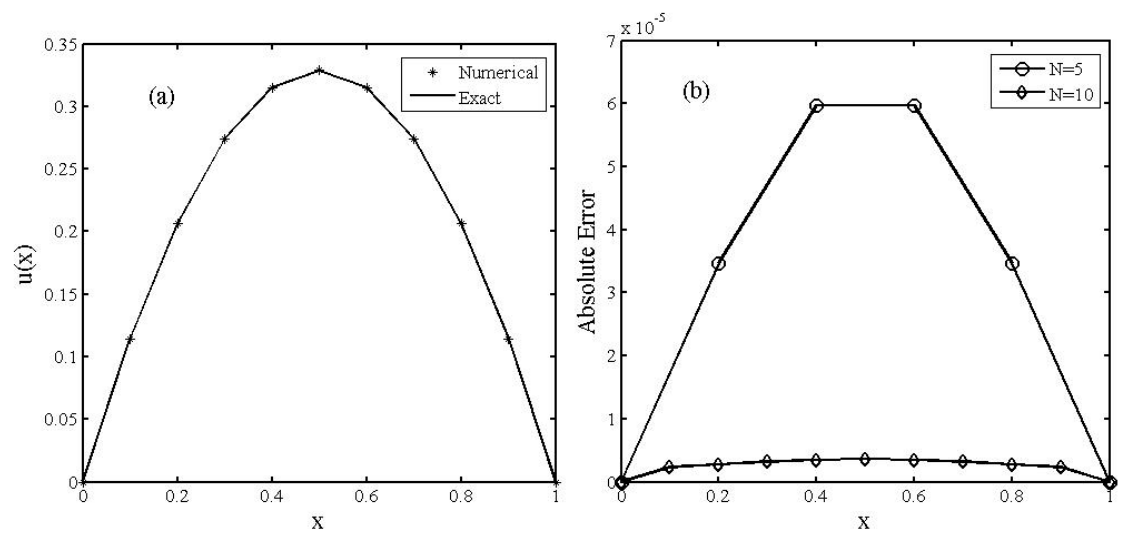

Figure 5. (a) Comparison of approximate values and exact values for Problem 5.5.

(b) Error graph for Problem 5.5 at different values of N (Table 9, col. III).

Problem 5.5. Consider the non-linear BVP (Bratu Problem)

$$
u^{(2)}(x)+2 e^{u(x)}=0, \quad 0<x<1 ; \quad u(0)=u(1)=0 .
$$

The theoretical solution of (5.9) is

$$
u(x)=-2 \ln (\cosh (1.17878(x-0.5))) / \cosh (0.589388) .
$$


Table 9. Comparison of MAE for the solution of Problem 5.5 at $N=10$

\begin{tabular}{lllll}
\hline $\begin{array}{l}\text { Our method for } \\
k=0\end{array}$ & $\begin{array}{l}\text { Our } k \text {-based } \\
\text { method }\end{array}$ & $\begin{array}{l}\text { Our fourth or- } \\
\text { der method }\end{array}$ & LGSM [1] & $\begin{array}{l}\text { Quintic } \\
\text { spline [7] }\end{array}$ \\
\hline $8.83 \times 10^{-4}$ & $3.56 \times 10^{-5}$ & $3.64 \times 10^{-6}$ & $5.7 \times 10^{-6}$ & $6.22 \times 10^{-6}$ \\
\hline $\begin{array}{l}\text { B-Spline } \\
\text { method [18] }\end{array}$ & $\begin{array}{l}\text { Quartic spline } \\
\text { method [6] }\end{array}$ & $\begin{array}{l}\text { Cubic } \\
\text { spline[20] }\end{array}$ & LADM [35] & ADM [22] \\
\hline $5.29 \times 10^{-5}$ & $1.10 \times 10^{-4}$ & $6.26 \times 10^{-4}$ & $1.24 \times 10^{-2}$ & $1.52 \times 10^{-2}$ \\
\hline
\end{tabular}

Abbreviations: ADM: Adomian Decomposition Method;

LGSM: Lie-group shooting method;

LADM: Laplace Adomian Decomposition Method

Problem 5.6. Consider the non-linear BVP

$$
u^{(2)}(x)=\frac{1}{2}(1+x+u)^{3}, \quad 0<x<1 ; \quad u(0)=u(1)=0 .
$$

The theoretical solution of (5.11) is

$$
u(x)=\frac{2}{(2-x)}-x-1 .
$$

The other non-linear BVPs mentioned in Problems 5.5 and 5.6, are also solved just like Problem 5.4 using Newton's method. Obtained results show the efficiency and accuracy of our proposed methods. Maximum absolute errors at the nodal points with a comparison with other methods are summarized in Table 9 for Problem 5.5 and in Table 10 for Problem 5.6, respectively. Figures 4-6 demonstrate the fourth order method solution and error graphs for nonlinear Problems 5.4-5.6 respectively with comparison of errors at the nodal points.

Table 10. Comparison of MAE for Problem 5.6 with Approaching spline method

$$
\text { at } N=5
$$

\begin{tabular}{lcccccc}
\hline \multicolumn{1}{c}{$x$ values } & 0 & 0.2 & 0.4 & 0.6 & 0.8 & 1 \\
\hline Our method for $k=0$ & 0 & $1.30 \times 10^{-3}$ & $2.40 \times 10^{-3}$ & $3.10 \times 10^{-3}$ & $2.80 \times 10^{-3}$ & 0 \\
Our $k$-based method & 0 & $2.70 \times 10^{-5}$ & $5.25 \times 10^{-5}$ & $7.19 \times 10^{-5}$ & $6.49 \times 10^{-5}$ & 0 \\
Our fourth order method & 0 & $3.80 \times 10^{-5}$ & $7.26 \times 10^{-5}$ & $9.92 \times 10^{-5}$ & $9.96 \times 10^{-5}$ & 0 \\
Approaching spline [31] & 0 & $1.40 \times 10^{-4}$ & $2.60 \times 10^{-4}$ & $3.20 \times 10^{-4}$ & $2.70 \times 10^{-4}$ & 0 \\
\hline
\end{tabular}



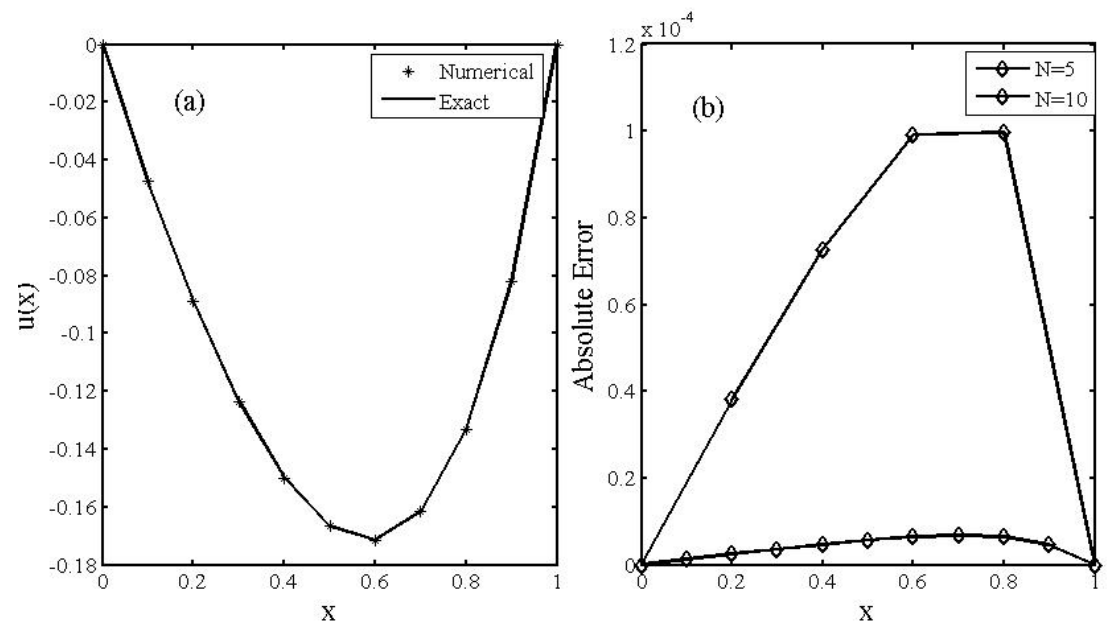

Figure 6. (a) Comparison of approximate values and exact values for Problem 5.6. (b) Error graph for Problem 5.6 at different values of N (Table 10).

\section{Conclusion}

A unique approach based on a different combination of non-polynomial cubic splines is used to develop various orders methods for solving linear and non-linear second order BVPs. We have also developed a parameter $k$-based method for smooth approximation of these BVPs. The convergence of the developed method is also established. Competence of the demonstrated technique can also be weighed through comparisons with the literature given in tables, which show that our results are comparatively better with more precise result. Graphs are plotted at different values of $N$ for all the problems, which clearly show that absolute errors decrease rapidly as step size $N$ increases.

Acknowledgment. The authors would like to acknowledge the many valuable discussions they had with Dr. Puneet Rana, Asst. Prof., JIIT, Noida, U.P., India during the development of this work and thank him for his valuable suggestions and technical help.

\section{References}

[1] Abbasbandy, S., Hashemi, M.S., Liu, C.S., The Lie-group shooting method for solving the Bratu equation, Commun. Nonlinear Sci. Numer. Simul., 16(2011), no. 11, 4238-4249.

[2] Ahlberg, J.H., Ito, T., A collocation method for two-point boundary value problems, Math. Comp., 29(1975), no. 131, 761-776.

[3] Ahlberg, J.H., Nilson, E.N., Walsh, J.L., The Theory of Splines and Their Applications, Academic Press, no. 38, New York/London, 1967.

[4] Akram, G., Tariq, H., Quintic spline collocation method for fractional boundary value problems, J. Assoc. Arabic Univ. Basic Appl. Sci., 23(2017), 57-65. 
[5] Albasiny, E.L., Hoskins, W.D., Cubic spline solutions to two-point boundary value problems, Comput. J., 12(1969), 151-153.

[6] Alayed, O.H., Ying, T.Y., Saaban, A., New fourth order quartic spline method for solving second order boundary value problems, Mathematika, 31(2015), 149-157.

[7] Alayed, O.H., Ying, T.Y., Saaban, A., Quintic spline method for solving linear and nonlinear boundary value problems, Sains Malays., 45(2016), no. 6, 1007-1012.

[8] Al-Said, E.A., Spline solutions for system of second order boundary value problems, Int. J. Comput. Math., 62(1996), 143-154.

[9] Al-Said, E.A., Quadratic spline solution of two point boundary value problems, J. Nat. Geom., 12(1997), 125-134.

[10] Al-Said, E.A., Cubic spline method for solving two-point boundary-value problems, Korean J. Comput. Appl. Math., 5(1998), 669-680.

[11] Al-Said, E.A., Spline methods for solving system of second order boundary-value problems, Int. J. Comput. Math., 70(1999), 717-727.

[12] Al-Said, E.A., The use of cubic splines in the numerical solution of a system of secondorder boundary value problems, Comput. Math. Appl., 42(2001), 861-869.

[13] Al-Said, E.A., Quartic splines solutions for a system of second order boundary value problems, Appl. Math. Comput., 166(2005), 254-264.

[14] Al-Said E.A. et al., Quartic spline method for solving second-order boundary value problems, Int. J. Phys. Sci., 6(2011), 4208-4212.

[15] Bickley W.G., Piecewise cubic interpolation and two-point boundary problems, Comput. J., 11(1968), 206-208.

[16] Blue, J.L., Spline function methods for nonlinear boundary value problems, Comm. ACM, 12(1969), no. 6, 327-330.

[17] Bongsoo, J., Two-point boundary value problems by the extended Adomian decomposition method, J. Comput. Appl. Math., 219(2008), 253-262.

[18] Caglar, H. et al., B-spline method for solving Bratu's problem, Int. J. Comput. Math., 87(2010), no. 8, 1885-1891.

[19] Chaurasia A., Srivastava P.C., Gupta Y., Spline based computational techniques for second, third and fourth order boundary value problems, Global J. Pure Appl. Math., 11(2015), 3203-3215.

[20] Chawla, M.M., Subramanian R., A new fourth-order cubic spline method for secondorder nonlinear two-point boundary-value problems, J. Comput. Appl. Math., 23(1988), $1-10$.

[21] Chen, F., Wong, P.J.Y., Discrete cubic spline method for second-order boundary value problems, Int. J. Comput. Math., 91(2013), 1041-1053.

[22] Deeba, E., Khuri, S.A., Xie, S., An algorithm for solving boundary value problems, J. Comput. Phys., 159(2000), 125-138.

[23] Fyfe, D.J., The use of cubic splines in the solution of two-point boundary value problems, Comput. J., 12(1969), 188-192.

[24] Henrici, P., Discrete Variable Methods in Ordinary Differential Equations, John Wiley, New York, 1961.

[25] Islam, M.S., Shirin, A., Numerical solutions of a class of second order boundary value problems on using Bernoulli polynomials, Appl. Math., 2(2011), 1059-1067. 
[26] Islam, S.U. et al., Quadratic non-polynomial spline approach to the solution of a system of second-order boundary-value problems, Appl. Math. Comput., 179(2006), 153-160.

[27] Islam, S.U., Tirmizi I.A., Non-polynomial spline approach to the solution of a system of second-order boundary-value problems, Appl. Math. Comput., 173(2006), 1208-1218.

[28] Jain, M.K., Aziz, T., Cubic spline solution of two-point boundary value problems with significant first derivatives, Comput. Methods Appl. Mech. Engrg., 39(1983), no. 1, 8391.

[29] Justine, H., Chew, J.V.L., Sulaiman, J., Quartic non-polynomial spline solution for solving two-point boundary value problems by using conjugate gradient iterative method, J. Appl. Math. Comput. Mech., 16(2017), 41-50.

[30] Justine, H., Sulaiman, J., Cubic non-polynomial solution for solving two-point boundary value problems using SOR iterative method, Trans. Sci. Technol., 3(2016), 469-475.

[31] Kalyani, P., Rama Chandra Rao, P.S., Solution of boundary value problems by approaching spline techniques, Int. J. Engrg. Math., 2013(2013), 1-9.

[32] Khalifa, A.K., Eilbeck, J.C., Collocation with quadratic and cubic splines, IMA J. Numer. Anal., 2(1982), 111-121.

[33] Khan, A., Parametric cubic spline solution of two point boundary value problems, Appl. Math. Comput., 154(2004), 175-182.

[34] Khan, A., Aziz, T., Parametric cubic spline approach to the solution of a system of second-order boundary-value problems, J. Optim. Theory Appl., 118(2003), 45-54.

[35] Khuri, S.A., A new approach to Bratu's problem, Appl. Math. Comput., 147(2004), 131136.

[36] Noor, M.A., Khalifa, A.K., Cubic splines collocation methods for unilateral problems, Internat. J. Sci. Engrg., 25(1987), 1527-1530.

[37] Raghavarao, C.V., Sanyasiraju, Y.V.S.S., Suresh, S., A note on application of cubic splines to two-point boundary value problems, Comput. Math. Appl., 27(1994), 45-48.

[38] Ramadan, M.A., Lashien, I.F., Zahra, W.K., Polynomial and non-polynomial spline approaches to the numerical solution of second order boundary value problems, Appl. Math. Comput., 184(2007), 476-484.

[39] Rashidinia, J., Jalilian, R., Spline solution of two point boundary value problems, Appl. Comput. Math., 9(2010), no. 2, 258-266.

[40] Rashidinia, J., Mohammadi, R., Jalilian, R., Cubic spline method for two-point boundary value problems, IUST Internat. J. Engrg. Sci., 19(2008), 39-43.

[41] Sakai, M., Piecewise cubic interpolation and two-point boundary value problems, Publ. RIMS, Kyoto Univ., 7(1972), 345-362.

[42] Sakai, M., Usmani, R.A., Quadratic spline and two point boundary value problems, RIMS, Kyoto Univ., 19(1983), 7-13.

[43] Srivastava, P.K., Kumar, M., Mohapatra, R.N., Quintic non-polynomial spline method for the solution of a second order boundary-value problem with engineering applications, Comput. Math. Appl., 62(2011), 1707-1714.

[44] Taiwo, O.A., Exponential fitting for the solution of two-point boundary value problems with cubic spline collocation tau-method, Int. J. Comput. Math., 79(2002), 299-306.

[45] Taiwo, O.A., Numerical solution of second order ordinary differential equations by spline collocation Tau method, Nigerian J. Math. Assoc., 6(1993), 1-16. 
[46] Usmani, R.A., Spline solutions for nonlinear two point boundary value problems, Int. J. Math. Math. Sci., 3(1980), 151-167.

[47] Usmani, R.A., Sakai, M., A connection between quartic spline solution and numerov solution of a boundary value problem, Int. J. Comput. Math., 26(1987), 263-273.

[48] Usmani, R.A., Warsi, S.A., Quintic spline solutions of boundary value problems, Comput. Math. Appl., 6(1980), no. 2, 197-203.

[49] Zahra, W.K., Numerical solutions for boundary value problems for ordinary differential equations by using spline functions, M. Sc. Diss., Tanta University, 2004.

[50] Zahra, W.K. et al., Cubic non-polynomial spline approach to the solution of a second order two-point boundary value problem, J. Amer. Sci., 6(2010), 297-302.

Anju Chaurasia

Department of Mathematics, Birla Institute of Technology,

Allahabad-211009 (U.P.), India

e-mail: anjuchaurasiya@rediffmail.com

Yogesh Gupta

Department of Mathematics, Jaypee Institute of Information Technology,

Noida-201307, India

e-mail: yogesh4july@gmail.com

Prakash C. Srivastava

Department of Mathematics, Birla Institute of Technology,

Patna-800014 (Bihar), India

e-mail: prakash_bit123@rediffmail.com 